Original Research Article

\title{
Howell's cytological grading of breast carcinomas - comparison with histological grading and its association with lymph node metastasis
}

\author{
Koshalya R. ${ }^{1}$, Muthu S. ${ }^{2}$, Shivashekar G. ${ }^{3}$ \\ ${ }^{1}$ Dr. Koshalya Rajendran, Post-graduate Student, ${ }^{2}$ Dr. Muthu Sudalaimuthu, Associate Professor, ${ }^{3}$ Dr. Shivashekar \\ Ganapathy, Professor and Head, Department of Pathology, SRM Medical College Hospital and Research Centre, \\ Kattankulathur, Kancheepuram District, Tamil Nadu, India.
}

Corresponding Author: Dr. Muthu Sudalaimuthu, Associate Professor, Department of Pathology, SRM Medical College Hospital and Research Centre, Kattankulathur, Kancheepuram District, Tamil Nadu, India. E-mail: drmuthus@gmail.com

\begin{abstract}
Background: Determining histological grade of breast carcinomas before mastectomy is necessary to decide about neoadjuvant chemotherapy. Grade obtained from fine needle aspiration cytology samples will help in such situations particularly when core needle biopsy is not done routinely as inresource-poor setups. Methods for doing such gradingare still under study. We performedgrading of breast carcinomas in Fine Needle Aspiration smearsby Howell's method and correlatedthe resultswith modified Bloom-Richardson histological grade in mastectomy specimens. For the first time, we also studied the prognostic significance of Howell's grade by studying its association with lymph node metastasis. Materials and methods: Fifty cases of Invasive carcinoma- no special type of breast in which both mastectomy and fine needle aspiration cytology were done between 2013 and 2015 were included in our study. Howell's grading was done in Papanicolaou, Haematoxylin \& eosin and May-Grunwald Giemsa stained cytology smears and correlated with modified Bloom Richardson histological grading in mastectomy specimens. Results: The two methods had same grade in 34 cases $(68 \%)$ and a kappa agreement value of 0.505 . They showed a good positive correlation (Spearman correlation coefficient 0.732 ) and significant association (chi-square test, p-0.0001). 12 cases were under-graded and four were over-graded. Lymph node metastasis significantly increased with increase in Howell's cytological grade (p-0.018). Conclusion: Howell's cytological grading is a simple method to grade breast carcinomas before mastectomy. This method has a good concordance with histological grading.The strong association with lymph node metastasis indicates the prognostic significance of this grading method.
\end{abstract}

Keywords- Breast carcinoma, Histological grading, Fine needle aspiration cytology, cytological grading, lymph node metastasis

\section{Introduction}

Breast cancer is the most common cancer among females globally as well as in India [1]. Histological grading is usually done in breast carcinomas by Nottingham modification of Bloom Richardson (NBR) grading method and is an important prognostic factor $[2,3]$. Apart from being a prognostic factor, grading also helps in choosing between the different treatment options available for breast carcinoma [4,5].

Manuscript received: $26^{\text {th }}$ August 2018

Reviewed: $4^{\text {th }}$ September 2018

Author Corrected: $10^{\text {th }}$ September 2018

Accepted for Publication: $14^{\text {th }}$ September 2018
Hence it is essential to obtain the grade of breast carcinoma early at the time of diagnosis itself. Although core needle biopsy can be used for this purpose, be

it is not done routinely in the resource-poor setup [5]. Hence it will be helpful if the grading of breast carcinomas can obtained earlier from the Fine Needle Aspiration Cytology (FNAC) samples it self. Although various methods have been suggested for cytological grading of breast carcinomas, a recent review by Bansal et al had recommended Howell's modification of NBR grading method to grade breast carcinomas in FNAC samples [6,7]. 


\section{Original Research Article}

Hence this study aimed at grading invasive carcinomano special type of breast in FNAC samplesby Howell's method and compared it with NBR histological grading obtained from mastectomy specimens in the same cases. We also studied the prognostic significance of Howell's grading method by studying its association with lymph node metastasis. To our knowledge, this aspect has not been studied in the past.

\section{Materials and Methods}

Place and Type of study- This study was done in the central laboratory of a tertiary health care hospital in South India by retrospectively studying cases of invasive carcinoma-no special type of breast diagnosed between 2013 and 2015.

Inclusion criteria- Cases of invasive carcinoma-no special type of breast diagnosed between 2013 and 2015 having both FNAC smears and mastectomy specimens were included in our study.

Exclusion criteria- Cases in which mastectomy were done after chemotherapy or radiotherapy were excluded from our study. This is because such therapies could have changed the grade of the tumor. Cases with inadequate material and those cases in which the slides/ blocks were not available were also excluded.

Sample collection and Sampling methods- Fine Needle Aspiration was performed using a $20 \mathrm{ml}$ disposable syringe and 22 gauge needle using aspiration technique with multi-directional passes. FNAC smears stained with Papanicolaou, Haematoxylin \& Eosin (H\&E) and May-Grunwald Giemsa stains were used for cytological grading by Howell's modification of NBR method. Sections from formalin fixed paraffin embedded tissue blocks prepared from tumour in mastectomy specimens were stained with $H \& E$ and used for histological grading by NBR method.

Grading procedure- Since mitoses and tubules are difficult to identify in FNAC smears, Howell had modified the criteria to grade breast carcinomas in FNAC smears [7].

Both microacini as well as branching elongated three dimensional tubular structures were included as tubule formation in cytologicalgrading. Mitotic count threshold for each score was also reduced. Howell's modified criteria are given in Table 1. Criteria used in NBR histological grading is given in Table 2 . Metaphase, anaphase and telophase mitotic figures were included for mitotic count. Olympus CX21 microscope with field view number 18 and field diameter $0.45 \mathrm{~mm}$ for the high power view was used. Sections from axillary lymph node were examined for metastasis.

Statistical methods- Statistical analysis was done using SPSS software version 21.0. Association between the two grading systems and association of Howell's grade with lymph node metastasis wereassessed using Chisquare test.

Kappa value of agreement was used to measure the strength of agreement between the two grading systems. Correlation between the two grading systems was assessed by Spearman rank correlation coefficient. $p$ value less than 0.05 was considered statistically significant.

\section{Results}

Fifty cases were included in our study based on the inclusion and exclusion criteria. Grade II was the most common NBR histological grade in our study (21 cases, 42\%) followed by grade III (15 cases, 30\%) and grade I (14 cases, $28 \%)$. Cytological grading was also done by assessing the three parameters tubule formation, pleomorphism and mitotic count (Figs 1-3). In cytological grading also grade II was the most common (25 cases, 50\%). 


\section{Original Research Article}

However grade I was the next most common grade in this method (16 cases, 32\%) followed by grade III ( 9 cases, $18 \%)$. 12 of the 14 histological grade I cases (85.7\%), 15 of the 21 histological grade II cases (71.4\%) and 7 of the 15 histological grade III cases (46.7\%) were graded similarly in the Howell's cytological method (Table 3 ). Thus the concordance was maximum in the grade I cases and least in the grade III cases.

The overall concordance was $68 \%$ with a kappa agreement value of 0.505 . The two methods showed good positive correlation in Spearman rank correlation test with a rho value of 0.732. There was a strong association between these two grading methods in chi-square test with a p value of 0.0001 . Overall 12 cases $(24 \%)$ were under-graded and 4 cases ( $8 \%$ ) were over-graded in the Howell's cytological grading method.

Thus under-grading was more common than over-grading in Howell's method. Among the total 50 cases, 24 cases (48\%) had lymph node metastasis. Five out of the 16 Howell's grade I cases (31.3\%), 11 out of the 25 Howell's grade II cases (44\%) and eight out of the nine Howell's grade III cases (88.9\%) had lymph node metastasis.

Thus the incidence of lymph node metastasis increased with increase in Howell's grade and this association was found to be statistically significant in chi-square test with a $\mathrm{p}$ value of 0.018 .

Table-1: Howell's modified criteria for Bloom-Richardson grading in cytology [7]

\begin{tabular}{|c|c|c|c|}
\hline $\begin{array}{c}\text { Parameter } \\
\text { To be assessed }\end{array}$ & Score 1 & Score 2 & Score 3 \\
\hline Tubule formation & $\begin{array}{c}\text { Tubule formation in } \\
>75 \% \text { of the tumor }\end{array}$ & $\begin{array}{c}\text { Tubule formation in } 10 \text { to } \\
75 \% \text { of the tumor }\end{array}$ & $\begin{array}{c}\text { Tubule formation in }<10 \% \\
\text { of the tumor }\end{array}$ \\
\hline $\begin{array}{c}\text { Nuclear pleomorphism } \\
\text { Migh power field }\end{array}$ & $\begin{array}{c}\text { Mild with small, regular } \\
\text { and uniform cells }\end{array}$ & Nuclei with moderate & Nuclei with marked \\
variation in size and shape & variation in size and shape \\
\hline Mitotic count per 10 & $0-1$ & $2-4$ & $>5$ \\
\hline
\end{tabular}

*Mitotic count scoring depends on the field diameter of the microscope. Values given here are for the microscope that we used with field diameter $0.45 \mathrm{~mm}$.

Grade 1 -Total score 3 to 5, Grade 2 -Total score 6 to 7 . Grade 3 -Total score 8 to 9 


\section{Original Research Article}

Table- 2: Nottingham modification of Bloom-Richardson histological grading [2]

\begin{tabular}{|c|c|c|c|}
\hline Parameter & Score 1 & Score 2 & Score 3 \\
\hline Tubule formation & $\begin{array}{l}\text { Tubule formation in } \\
>75 \% \text { of the tumor }\end{array}$ & $\begin{array}{c}\text { Tubule formation in } 10 \text { to } \\
75 \% \text { of the tumor }\end{array}$ & $\begin{array}{c}\text { Tubule formation in }<10 \% \\
\text { of the tumor }\end{array}$ \\
\hline Nuclear pleomorphism & $\begin{array}{l}\text { Mild with small, regular } \\
\text { and uniform cells }\end{array}$ & $\begin{array}{c}\text { Nuclei with moderate } \\
\text { variation in size and shape }\end{array}$ & $\begin{array}{c}\text { Nuclei with marked } \\
\text { variation in size and shape }\end{array}$ \\
\hline $\begin{array}{l}\text { Mitotic count per } 10 \\
\text { High power field* }\end{array}$ & $0-5$ & $6-10$ & $>10$ \\
\hline
\end{tabular}

* Mitotic count scoring depends on the field diameter of the microscope. Values given here are for the microscope that we used with field diameter $0.45 \mathrm{~mm}$.

Grade 1 -Total score 3 to 5, Grade 2 -Total score 6 to 7. Grade 3 -Total score 8 to 9

Table-3: Comparison of Howell's cytological grade with NBR histological grade.

\begin{tabular}{|c|c|c|c|c|}
\hline \multirow{2}{*}{ Cytological grade } & \multicolumn{3}{|c|}{ Histological grade } \\
\cline { 2 - 5 } & Grade I & Grade II & Grade III & Total \\
\hline Grade I & 12 & 4 & 0 & 25 \\
\hline Grade II & 2 & 15 & 8 & 9 \\
\hline Grade III & 0 & 2 & 7 & 5 \\
\hline Total & 14 & 21 & & 5 \\
\hline
\end{tabular}




\section{Original Research Article}

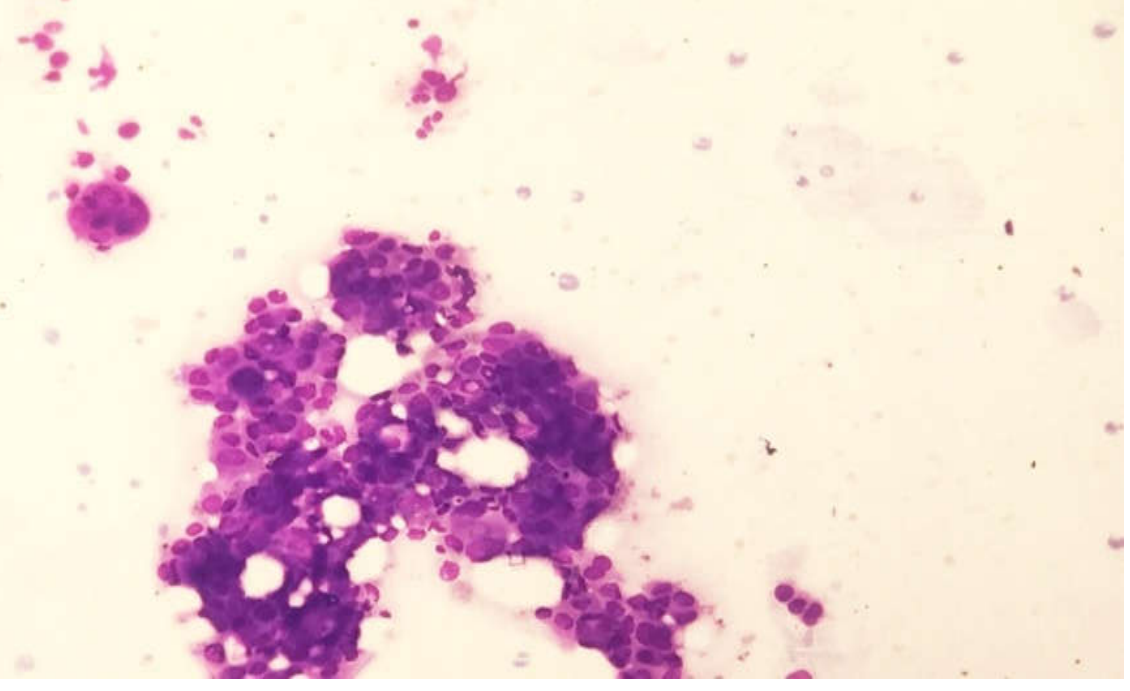

Fig-1: Tubular arrangement of tumour cells (May-Grunwald Giemsa, X100)

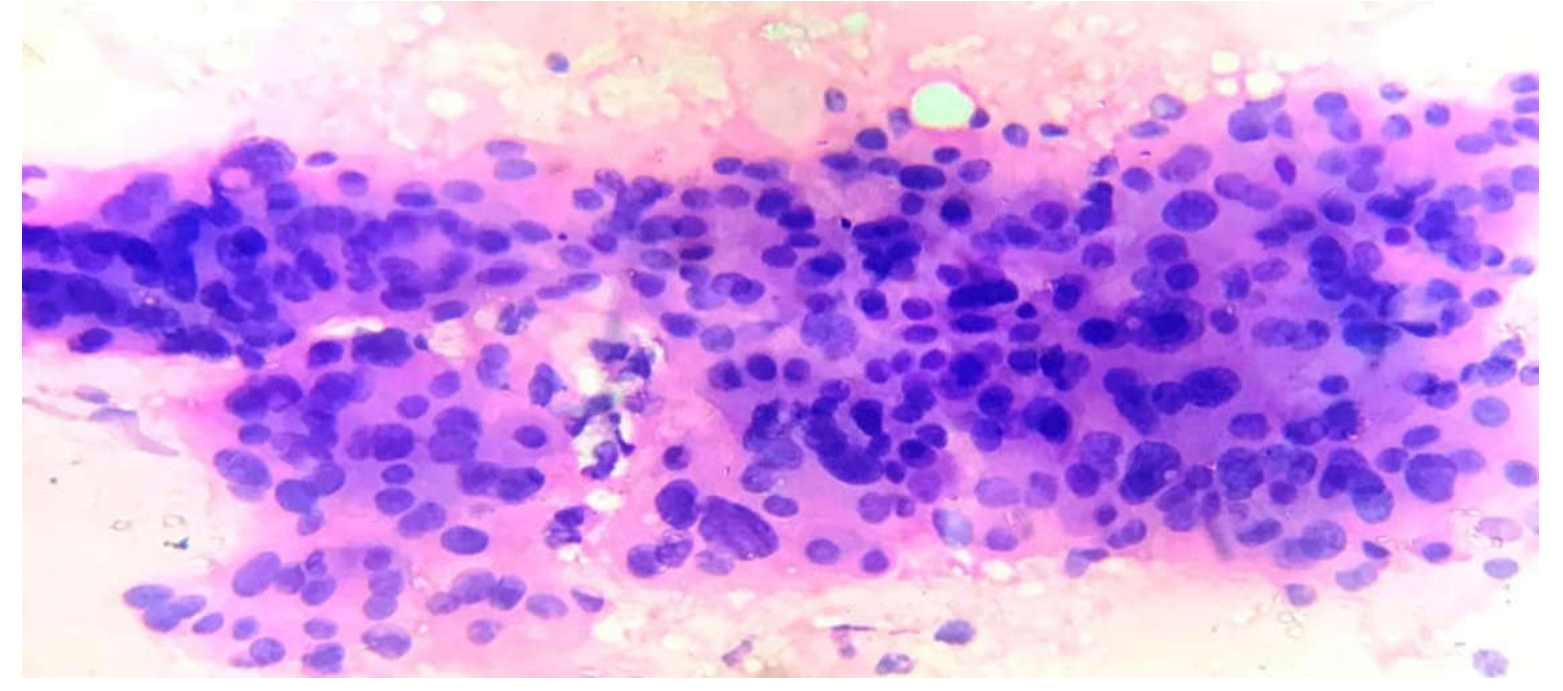

Fig-2: Tumour cells showing marked pleomorphism (H\&E, X400)

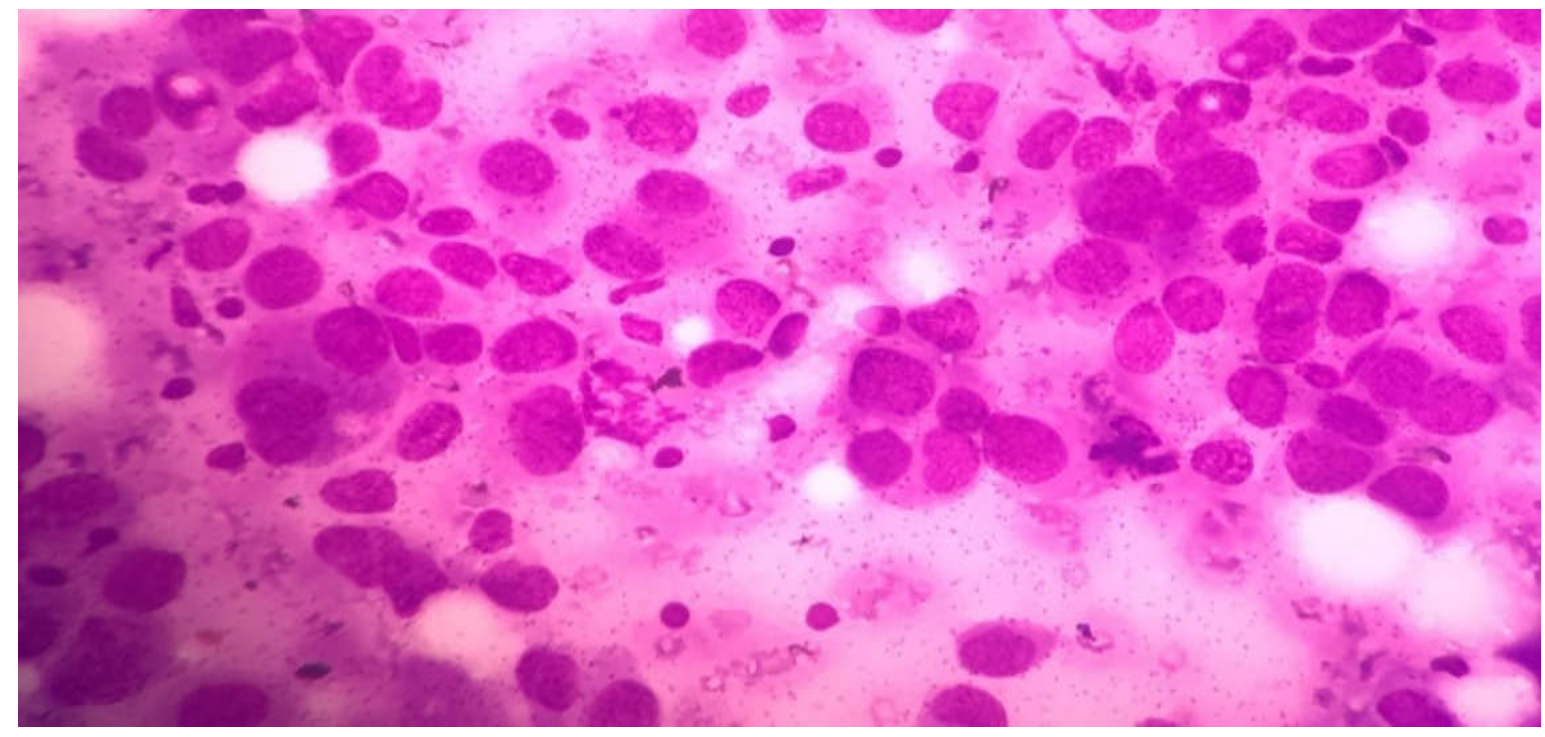

Fig-3: Mitotic figure seen among tumour cells (May-Grunwald Giemsa, X400) 


\section{Original Research Article}

\section{Discussion}

Histological grade of breast carcinoma has got prognostic value and is routinely done in almost every case. Apart from being a prognostic marker, grade of breast carcinoma also helps in deciding treatment as high grade tumours respond well to neo-adjuvant/ adjuvant chemotherapy whereas low grade tumours do not [4]. Histological grade of the tumour should be ideally obtained from excision samples or mastectomy specimens.

But to decide about neo-adjuvant chemotherapy, grading has to be done initially itself before mastectomy. In such situations, the pre-treatment samples obtained by FNAC or core needle biopsy are usually the only available materials for grading. In resource-poor setup, core needle biopsy might not be done routinely in all cases, particularly if the diagnosis of breast carcinoma has already been made from FNAC samples. In such situations, grading in FNAC smears becomes crucial.

Also in situations when neo-adjuvant chemotherapy has been already given in the patient, grading done on mastectomy specimens after chemotherapy might not

reflect the actual grade of the tumour [8,9]. In such situations FNAC samples obtained initially at the time of diagnosis, before initiating chemotherapy, will help in obtaining the original grade of the tumour. Thus there is a need for a method to grade breast carcinomas in FNAC smears itself. Grading of breast carcinomas is usually done in histopathology by NBR method. However the same method cannot be used for grading

of breast carcinomas in FNAC smears as mitoses and tubule formation are usually low in FNAC smears than in the histopathology sections [7].

Hence many new methods have been suggested for grading of breast carcinomas in FNAC smears $[6,10$
14]. Howell et al suggested a method in which they made modifications in NBR grading system so that the new method can be used for grading in cytology smears [7].

They made modifications in the criteria to score tubules and mitotic count. The advantage with Howell's method is that all the parameters assessed in histological grading are assessed in this method also. Thus it almost resembles the NBR histological grading method. But the other cytological grading methods do not assess all these three parameters.

A review by Bansal et al claimed this method to be superior to the other methods proposed for grading breast carcinomas in cytology smears [6]. In our study Howell's cytological grading method had a concordance of $68 \%$ with NBR histological grading method. This is slightly higher than the previous studies by Saha et al (63.16\%) and Howell et al $(57.1 \%)[7,15]$.

Walke et al had a concordance rate of $68 \%$ similar to our study [16]. Einstein et al and Arul et al had slightly higher concordance rates of $69.4 \%$ and $74.5 \%$ respectively $[17,18]$. Thus most studies have reported concordance rate around the range of $60-75 \%$ in which our results also fit in. The kappa value of agreement between the two grading methods, which is a better index than simply calculating the percentage of agreement (as it adjusts for the agreement that takes place by chance) was 0.505 in our study. This was higher than kappa value of 0.450 noted in the study by Walke et al, 0.40 noted by Saha et al, 0.436 noted by Einstein et al and 0.485 noted by Arul et al[15-18]. Spearman rank correlation coefficient was also higher in our study (0.732) compared to the previous studies (range from 0.614 to 0.674$)[15,17,18]$.

Careful examination of the FNAC smears for identifying the most pleomorphic areas and areas with highest mitotic count helped in scoring these parameters correctly and obtaining the correct grade of the tumour. 


\section{Original Research Article}

None of the cases were discordant by more than one grade in our study similar to the results of Walke et al [16].

Concordance rate was high in grade I tumours (85.7\%) and none of the histological grade III cases were reported as grade I in Howell's cytological grading. Thus Howell's method helps in identifying the low grade cases which are least likely to respond to chemotherapy. If this can be proved clinically, Howell's grading method will help to avoid the cost and adverse effects associated with neo-adjuvant chemotherapy in patients who are least likely to respond to it.

When we analysed the discordant cases, under-grading (12 cases) was more common than over-grading (4 cases) in Howell's method in our study. Detailed description of the discordant cases as to whether they were under-graded or over-graded have not been described in detail in most of the abovementionedstudies. In the study by Walke at al, overgrading (27 cases) was little more common than undergrading (21cases)[16].

However Howell et al mention in their article that discordance in grading was noted mainly in the high grade cases indicating that under-grading was common in their study as well [7].

Similarly in the study by Saha at al the number of grade III cases in Howell's method (16 cases) was only around half the number of grade III cases in histological grading (29 cases) indicating that undergrading was common in their study as well [15]. In our study also, concordance was least for the histological grade III cases $(46.7 \%)$.

Thus under-grading seems to be more common than over-grading in Howell's method and was more common in the high grade tumours in our study. Although seen in a limited number of cases, this can be considered as a minor disadvantage of this method. We further observed that mitotic count score was decreased in most of these under-graded cases and was the major reason for this under-grading. This might be either because mitotic figures are fragile and get degenerated while making FNAC smears or because of the fact that only less number of cells are sampled in a FNAC compared to excision samples. This has been mentioned by Howell et al also in their article [7]. In fact this was the reason why they lowered the cutoff for the various mitotic count scores in their cytological grading method itself.

Further studies need to be done to identify whether any further modification of the mitotic count scoring will improve the agreement between the two methods even more.Another reason for under-scoring mitoses could be that mitoses are usually more at the growing edge of the tumour, which might not be sampled during FNAC procedure. Bansal et al showed in their study that mitoses score and hence the grade of the tumor can be more accurately assessed in Howell's method if FNAC is done under ultrasound guidance from the periphery of the tumour [5].

However, we didn't use ultrasound guidance to sample the periphery of the tumour. This was because we wanted to study the usefulness of Howell's method using a methodology which can be done cost-effectively even in resource-poor setups in future. To our knowledge, our study is the first to study the relationship between Howell's grade and lymph node metastasis. We found in our study that as the Howell's grade increased, the incidence of lymph node metastasis also increased. This association was found to be statistically significant as well (p-0.018). This indicates the prognostic significance of Howell's grading method as lymph node metastasis has got adverse prognosis. 


\section{Original Research Article}

\section{Conclusion}

Howell's cytological grading of breast carcinomas is a simple method which has a good concordance rate with histological grading. This method can help in grading breast carcinomas without any additional invasive procedures, particularly in resource-poor setup. This will help in identifying the low grade cases which usually do not respond well to chemotherapy. The strong association with lymph node metastasis indicates the prognostic significance of this grading method. These findings need to be confirmed by further large scale studies.

Contribution by different authors- Dr. Koshalya Rajendran contributed to study designing, literature search and review, data acquisition and analysis, statistical analysis, manuscript preparation and editing. Dr. Muthu Sudalaimuthu contributed to study designing, literature review, data acquisition and analysis, statistical analysis, manuscript preparation and editing. Dr. Shivashekar Ganapathy contributed to study designing, literature review, data analysis, manuscript preparation and editing.

Additional knowledge gained from the studyHowell's cytological grade showed concordance with NBR histological grade in a good number of cases. Discordance was noted mainly in the high grade tumours. A statistically significant association was noted between Howell's cytological grade and lymph node metastasis indicating the prognostic significance of this method.

Acknowledgements- We thank all our colleagues and technicians for their helps during the study.

Funding: Nil, Conflict of interest: None initiated

Permission from IRB: Yes

\section{References}

1. Malvia S, Bagadi SA, Dubey US, Saxena S. Epidemiology of breast cancer in Indian women. Asia
Pac J Clin Oncol. 2017 Aug;13(4):289-295. doi: 10. 1111 / ajco.12661. Epub 2017 Feb 9.

2. Elston CW, Ellis IO. Pathological prognostic factors in breast cancer. I. The value of histological grade in breast cancer: experience from a large study with long-term follow-up. Histopathology. 1991 Nov; 19 (5):403-10.

3. Schwartz AM, Henson DE, Chen D, Rajamarthandan S. Histologic grade remains a prognostic factor for breast cancer regardless of the number of positive lymph nodes and tumor size: a study of 161708 cases of breast cancer from the SEER Program. Arch Pathol Lab Med. 2014 Aug;138(8):1048-52. doi: 10.5858/arpa. 2013-0435-OA.

4. Amat S, Penault-Llorca F, Cure H, et al. ScarffBloom-Richardson (SBR) grading: a pleiotropic marker of chemosensitivity in invasiveductal breast carcinomas treated by neoadjuvant chemotherapy. Int J Oncol. 2002 Apr; 20(4):791-6.

5. Bansal C, Singh US, Misra S, et al. Comparative evaluation of the modified Scarff-Bloom-Richardson grading system on breast carcinoma aspirates and histopathology. Cytojournal. 2012; 9:4. doi: 10.4103/ 1742-6413.92550. Epub 2012 Jan 31.

6. Bansal C, Pujani M, Sharma KL, et al. Grading systems in the cytological diagnosis of breast cancer: a review. J Cancer Res Ther. 2014 Oct-Dec;10(4):839-45. doi: 10.4103/0973-1482.140979.

7. Howell LP, Gandour-Edwards R, O' Sullivan D. Application of the Scarff-Bloom-Richardson tumor grading system to fine-needle aspirates of the breast. Am J Clin Pathol. 1994 Mar;101(3):262-5.

8. Provenzano E, Pinder SE. Modern therapies and iatrogenic changes in breast pathology. Histopathology. 2017 Jan;70(1):40-55. doi: 10.1111/his.13098. 


\section{Original Research Article}

9. Moll UM, Chumas J. Morphologic effects of neoadjuvant chemotherapy in locally advanced breast cancer. Pathol Res Pract. 1997;193(3):187-96.

10. Mouriquand J, Gozlan-Fior M, Villemain D, et al. Value of cytoprognostic classification in breast carcinomas. J Clin Pathol. 1986 May;39(5):489-96.

11. Robinson IA, McKee $G$, Nicholson A, et al. Prognostic value of cytological grading of fine-needle aspirates from breast carcinomas. Lancet. 1994 Apr 16; 343 (8903):947-9.

12. Hunt CM, Ellis IO, Elston CW, et al. Cytological grading of breast carcinoma--a feasible proposition? Cytopathology. 1990;1(5):287-95.

13. Taniguchi E, Yang Q, Tang W, et al. Cytologic grading of invasive breast carcinoma. Correlation with clinicopathologic variables and predictive value of nodal metastasis. Acta Cytol. 2000 Jul-Aug;44(4):58791. doi:10.1159/000328533

14. Khan MZ, Haleem A, Al Hassani H, Kfoury H. Cytopathological grading, as a predictor of histopatho- logical grade, in ductal carcinoma (NOS) of breast, on air-dried Diff-Quik smears. Diagn Cytopathol.2003 Oct; 29 (4):185-93.

15. Saha K, Raychaudhuri G, Chattopadhyay BK, Das I. Comparative evaluation of six cytological grading systems in breast carcinoma. J Cytol. 2013 Apr; 30 (2):87-93. doi: 10.4103/0970-9371.112647.

16. Walke VA, Gunjkar G. Comparative evaluation of six parametric Robinson and three parametric Howell's modification of Scarf-Bloom Richardson grading method on breast aspirates with histopathology: A prospective study. Cytojournal. 2017 Dec 27;14:31. doi: 10. 4103/cytojournal.cytojournal_31_17.E Collection 2017. 17. Einstien D, Omprakash BO, Ganapathy H, Rahman S. Comparison of 3-tier cytological grading systems for breast carcinoma. ISRN Oncol. 2014 Mar 12; 2014: 252103. doi: 10.1155/2014/252103. E Collection 2014.

18. Arul P, Masilamani S. Comparative evaluation of various cytomorphological grading systems in breast carcinoma. Indian J Med Paediatr Oncol. 2016 Apr-Jun; 37(2):79-84. doi: 10.4103/0971-5851.180141.

\section{How to cite this article?}

Koshalya R, Muthu S, Shivashekar G. Howell's cytological grading of breast carcinomas - comparison with histological grading and its association with lymph node metastasis. Trop J Path Micro 2018; 4(5):377-383.doi:10.17511/jopm. 2018.i05.02 